\title{
Association of prenatal urinary phthalate metabolite concentrations and childhood BMI and obesity
}

Kim G. Harley ${ }^{1}$, Kimberly Berger ${ }^{1}$, Stephen Rauch ${ }^{1}$, Katherine Kogut ${ }^{1}$, Birgit Claus Henn ${ }^{2}$, Antonia M. Calafat ${ }^{3}$, Karen Huen ${ }^{1}$, Brenda Eskenazi ${ }^{1}$ and Nina Holland ${ }^{1}$

BACKGROUND: Although experiments in animals suggest that phthalates may have obesogenic effects, studies on prenatal exposure in children show inconsistent results.

METHODS: We measured urinary concentrations of 11 phthalate metabolites collected twice during pregnancy from mothers participating in the Center for the Health Assessment of Mothers and Children of Salinas (CHAMACOS) cohort study $(N=345)$. Height, weight, waist circumference, and percent body fat were assessed in their children between 5 and 12 years of age. We used generalized estimating equations to examine associations at each age and tested for interaction by sex.

RESULTS: Metabolites of diethyl phthalate (DEP), di-n-butyl phthalate (DBP), butyl benzyl phthalate, and di(2-ethylhexyl) phthalate (DEHP) were positively associated with BMl z-score, waist circumference $z$-score, and percent body fat at multiple ages. At age 12, we observed increased odds of being overweight/obese with each doubling of prenatal concentrations of DEP (odds ratio $=1.3$; $95 \%$ confidence intervals: 1.1, 1.4), $\operatorname{DBP}(1.2 ; 1.0,1.4)$, and $\operatorname{DEHP}(1.3 ; 1.0,1.6)$ metabolites. Results were similar in boys and girls except for DBP metabolites and the non-specific metabolite mono-(3-carboxypropyl) phthalate, which showed positive associations only in boys.

CONCLUSION: In utero exposure to certain phthalates is associated with increased BMl and risk for overweight/obesity in childhood.

$\mathbf{T}$ he prevalence of obesity in children and adults has increased worldwide in recent decades (1), such that $35 \%$ of men and $40 \%$ of women in the United States are currently obese (2). Evidence suggests that increased caloric intake and reduced physical activity may not be sufficient to explain the current obesity epidemic (3). Exposure to endocrinedisrupting chemicals (EDCs), including phthalates-a class of chemicals widely used in personal care products, fragrances, medication coatings, adhesives, and polychlorinated vinyl (PVC) plastics-has been hypothesized as a possible contributor to the etiology of obesity (4). The prenatal period may be a critical developmental window for exposure to "obesogenic" chemicals to impact BMI later in life (5).

Exposure to phthalates is almost ubiquitous, with metabolites of eight common phthalates (Table 1) detected in $94 \%$ or more of Americans participating in the 2001-2010 cycles of the National Health and Nutrition Examination Survey (NHANES) (6). Low-molecular-weight phthalates, including diethyl phthalate (DEP), di- $n$-butyl phthalate (DBP), and diisobutyl phthalate (DiBP), are used in personal care products as carriers for fragrance and in the coatings of some medications. High-molecular-weight phthalates, including butyl benzyl phthalate (BBzP), di(2-ethylhexyl) phthalate (DEHP), and di-isononyl phthalate (DiNP), are used to soften plastics, particularly PVC building materials. These phthalates break down into monoester metabolites that are eliminated in urine.

Several phthalates, including the metabolites of DBP, BBzP, and DEHP, have been shown to bind to and activate peroxisome proliferator-activated receptor-alpha (PPAR- $\alpha$ ) and -gamma (PPAR- $\gamma$ ), nuclear receptors involved in fatty acid oxidation, adipogenesis, and fat storage (7). DEHP has also been associated with reduced thyroid hormone levels in rodent studies, which may interrupt energy homeostasis and increase body fat (8). Much of the rodent research focuses on DEHP, whereas other phthalates have been less studied with regard to body weight. Studies on adult male (8) and female (9) mice show that exposure to DEHP at doses ranging from $0.05 \mathrm{mg} / \mathrm{kg}$ per day (similar to occupational exposure levels) to $500 \mathrm{mg} / \mathrm{kg}$ per day were associated with increased food intake, body weight, and visceral fat. Prenatal and lactational exposure to DEHP at doses ranging from 0.05 to $300 \mathrm{mg} / \mathrm{kg}$ per day has also been associated with increased body weight and fat mass $(9,10)$, which may not manifest until the animal reaches adulthood. For example, in male rat offspring, prenatal and lactational exposure of dams to $300 \mathrm{mg} / \mathrm{kg}$ per day of DEHP was associated with decreased body weight at birth but with significantly higher body weight at

\footnotetext{
${ }^{1}$ Center for Environmental Research and Children's Health (CERCH), School of Public Health, University of California, Berkeley, CA; ${ }^{2}$ Department of Environmental Health, Boston University School of Public Health, Boston, Massachusetts; ${ }^{3}$ Division of Laboratory Sciences, National Center for Environmental Health, Centers for Disease Control and Prevention, Atlanta, Georgia. Correspondence: Kim G. Harley (kharley@berkeley.edu)

Received 19 January 2017; accepted 4 April 2017; advance online publication 31 May 2017. doi:10.1038/pr.2017.112
} 
Table 1. Phthalates and their monoester metabolites

\begin{tabular}{lll}
\hline Phthalate & Used in & Urinary metabolite \\
\hline Diethyl phthalate (DEP) & Personal care products, fragrance & Monoethyl phthalate (MEP) \\
Di-n-butyl phthalate (DBP) & Personal care products, medications & Mono- $n$-butyl phthalate (MBP) \\
Di-isobutyl phthalate (DiBP) & Personal care products, medications \\
Benzylbutyl phthalate (BzBP) & $\begin{array}{l}\text { Polychlorinated vinyl (PVC), adhesives, } \\
\text { sealants, car care products }\end{array}$ & Mono-isobutyl phthalate (MiBP) \\
Di-2-ethylhexyl phthalate (DEHP) & $\begin{array}{l}\text { PVC, flexible plastics (toys, film packaging, medical } \\
\text { devices, building materials, garden hoses, and so on) }\end{array}$ & $\begin{array}{l}\text { Monobenzyl phthalate (MBzP) } \\
\text { Mono-(2-ethyl-5-hydroxyhexyl) phthalate (MEHHP) }\end{array}$ \\
& & $\begin{array}{l}\text { Mono-(2-ethyl-5-oxohexyl) phthalate (MEOHP) } \\
\text { Mono-(2-ethyl-5-carboxypentyl) phthalate (MECCP) }\end{array}$ \\
Di-n-octyl phthalate (DOP) & PVC, flexible plastics & Mono-(3-carboxypropyl) phthalate (MCPP) \\
Di-isononyl phthalate (DiNP) & PVC, flexible plastics & Mono-(carboxyoctyl) phthalate (MCOP) \\
Di-isodecyl phthalate (DiDP) & PVC, flexible plastics & Mono-(carboxynonyl) phthalate (MCNP)
\end{tabular}

Adapted from CDC National Biomonitoring Program, Phthalate Biomonitoring Summaries http://www.cdc.gov/biomonitoring/biomonitoring_summaries.html.

${ }^{a} \mathrm{MCPP}$ is also a minor metabolite of DBP and a non-specific metabolite of several high-molecular-weight phthalates.

puberty and adulthood in the exposed group compared with controls (10).

Cross-sectional studies in humans have also linked urinary metabolites of various phthalates to increased BMI, waist circumference, and obesity in children (11) and adults (12). However, only a few longitudinal studies have examined the associations of prenatal exposure and later BMI (13-17), and these studies have shown inconsistent results and often sexspecific associations. A study in France that examined only boys found that prenatal urinary concentrations of the main DEP metabolite were associated with increased BMI at age 5 years (17); however, three other studies have found prenatal phthalate metabolites to be associated with decreased BMI in boys (13-15). Specifically, a study on Spanish children found that urinary concentrations of high-molecular-weight phthalates in mothers during pregnancy were negatively associated with BMI $z$-score in boys at age 4 and 7 years but were positively (although non-significantly) associated with BMI $z$ score in girls at the same ages (16). Two different cohort studies in New York City also found negative associations of maternal urinary metabolites with BMI in boys $(14,15)$. A pooled study (13) of three US cohorts, including the two previously mentioned New York cohorts, found that prenatal urinary concentrations of mono-3-carboxypropyl phthalate (MCPP), a metabolite of DBP and of several high-molecularweight phthalates, were associated with increased risk for overweight/obesity in boys and girls at ages 4-7 years, whereas metabolites of DEP and DEHP were associated with decreased BMI $z$-scores in girls. In addition, a study that examined only girls found that urinary concentrations of lowmolecular-weight phthalates at ages $6-8$ years were associated with increased BMI and waist circumference at ages 7-13 years (18).

To further examine prenatal phthalate exposure and BMI in childhood, we assessed the association of maternal prenatal exposure with eight phthalates (described by concentrations of 11 phthalate metabolites in maternal urine) with BMI, waist circumference, and percent body fat in their children in a longitudinal birth cohort study. We examined anthropometric outcomes between 5 and 12 years of age, with a particular focus on age 12, as animal studies suggest that the obesogenic effects of phthalates may not manifest until puberty.

\section{METHODS}

\section{Study Population}

Study participants were mothers and children enrolled in the Center for the Health Assessment of Mothers and Children of Salinas (CHAMACOS), a longitudinal cohort study examining the effects of early life environmental exposures on children's growth, health, and development. The study is based in the Salinas Valley, an agricultural region of California with a large Mexican immigrant population. Recruitment and enrollment in the study were conducted in 19992000. Pregnant women receiving prenatal care at partnering clinics that served predominantly low-income, Latina patients were screened for eligibility and were invited to participate. Eligible women were at least 18 years of age, spoke English or Spanish, qualified for low-income health insurance (Medicaid), and planned to deliver at the county hospital.

Follow-up of this prospective cohort study is ongoing. Maternal interviews and child developmental assessments have been conducted every 1-2 years through early adolescence. A total of 601 women were enrolled during pregnancy, 537 remained in the study through delivery, and 365 children completed anthropometric measurements at least once between 5 and 12 years of age. For this analysis, we randomly dropped one twin from each pair of twins in the study $(N=3)$ to maintain independent observations. We excluded 17 children who were missing prenatal phthalate biomarker measurements, for a final sample size of 345. All study activities were approved by the Institutional Review Board (IRB) of the University of California, Berkeley. The Centers for Disease Control and Prevention (CDC) IRB deferred approval to the IRB at the University of California, Berkeley. Informed consent was obtained from all mothers; oral assent was obtained from children beginning at age 7 years and written assent was obtained at age 12 years.

\section{Data Collection}

The data for this analysis were collected from interviews with mothers near the end of the first and second trimesters of pregnancy and when their children were $5(N=313), 7(N=322), 9(N=321)$, $10 \frac{1}{2}(N=303)$, and $12(N=320)$ years of age. All interviews were 
conducted in English or Spanish using structured questionnaires. At the baseline interview, conducted near the end of the first trimester of pregnancy, mothers were asked their age, marital status, education level, number of years residing in the United States, and household income, as well as information about smoking, soda consumption, and drug and alcohol use during pregnancy. Information on pregnancy weight gain, gestational duration, and infant birth weight was gathered from medical records. We calculated pre-pregnancy BMI using measured height and self-reported pre-pregnancy weight, if available, or imputed based on weight at first prenatal care visit if pre-pregnancy weight was unknown or out of range $(n=39)$. At the interviews when the children were between 5 and 12 years of age, mothers were asked about dietary and physical activity behaviors of their children, including household food security and fast food consumption.

\section{Phthalate Biomarker Measurements}

Urine samples were collected from mothers at the time of the two pregnancy interviews (mean \pm SD: $14.0 \pm 4.8$ and $26.9 \pm 2.5$ weeks gestation). Samples were collected in polypropylene urine cups, aliquoted into glass vials, and stored at $-80^{\circ} \mathrm{C}$ until shipment to the CDC in Atlanta, Georgia, for analysis.

Samples were analyzed using solid-phase extraction coupled with isotope dilution high-performance liquid chromatographyelectrospray ionization-tandem mass spectrometry to quantify concentrations of the following 11 phthalate metabolites of 8 parent compounds (Table 1): monoethyl phthalate (MEP, a metabolite of DEP); mono- $n$-butyl phthalate (MBP, a metabolite of DBP); mono-isobutyl phthalate (MiBP, a metabolite of di-isobutyl phthalate (DiBP)); monobenzyl phthalate $(\mathrm{MBz}$, a metabolite of $\mathrm{BBzP})$; four metabolites of DEHP (mono-2-ethylhexyl phthalate (MEHP), mono(2-ethyl-5-hydroxyhexyl) phthalate (MEHHP), mono-(2-ethyl-5oxohexyl) phthalate (MEOHP), and mono-(2-ethyl-5-carboxypentyl) phthalate (MECCP)); mono(carboxyoctyl) phthalate (MCOP; a metabolite of di-isononyl phthalate (DiNP)); mono(carboxynonyl) phthalate (MCNP; a metabolite of di-isodecyl phthalate (DiDP)); and mono(3-carboxypropyl) phthalate (MCPP; a metabolite of several high-molecular-weight phthalates and a minor metabolite of DBP). Analytic methods have been published previously (19). Phthalate metabolite concentrations were reported in $\mathrm{ng} / \mathrm{ml}$ of urine. Limits of detection (LOD) ranged from 0.2 to $0.6 \mathrm{ng} / \mathrm{ml}$. Concentrations less than LOD were assigned the instrumental reading values, if available, or an imputed value less than LOD randomly selected from the lognormal distribution using maximum likelihood estimation (20), if not.

Urinary-specific gravity was measured using a hand-held refractometer (National Instrument Company Inc., Baltimore, Maryland). We corrected for urinary dilution using the formula (analyte concentration $\times 0.24) /($ sample specific gravity-1) (21). For 65 women missing specific gravity measures, urinary-specific gravity was imputed on the basis of urinary creatinine concentrations.

\section{Anthropometric Measurements}

At ages 5, 7, 9, 101/2, and 12 years, children were weighed and measured without shoes or jackets. Standing height was measured using a stadiometer (Seca 222, Chino, California) and waist circumference was measured using a measuring tape held at the level of the iliac crest. Height and waist circumference were measured in triplicate and averaged. Body weight $(\mathrm{kg})$ was measured using an electronic scale at ages 5 and 7 years (Tanita 1582, Arlington Heights, Illinois) and using a foot-to-foot bioimpedence scale (Tanita TBF $300 \mathrm{~A}$, Arlington Heights, Illinois) that provided both weight and percent body fat at ages $9,10^{1 / 2}$, and 12 years. BMI was calculated as weight $/$ height ${ }^{2}\left(\mathrm{~kg} / \mathrm{m}^{2}\right)$ and classified as underweight, normal weight, overweight, or obese according to the CDC's age- and sex-specific percentiles (22). Age- and sex-standardized BMI and waist circumference $z$-scores were calculated using CDC norms (23).

\section{Statistical Analysis}

Prenatal phthalate exposure was analyzed as the average of the two pregnancy phthalate metabolite concentration measures. Average phthalate metabolite concentrations were examined continuously as $\log _{2}$-transformed variables and categorically by quartiles. DEHP devolves to several highly correlated metabolites (including MEHP, MEHHP, MEOHP, and MECCP) that individually account for only a fraction of total DEHP exposure but may represent $>60 \%$ of the DEHP dose when taken together (24); thus, we summed the molar concentrations of MEHP, MEHHP, MEOHP, and MECCP to obtain a $\Sigma$ DEHP variable. All other metabolites were examined individually. Although other studies have also generated summed variables for low-molecular-weight (MEP, MBP, MiBP) and high-molecularweight (MBzP, $\Sigma \mathrm{DEHP}, \mathrm{MCPP}, \mathrm{MCOP}, \mathrm{MCNP}$ ) metabolites, we chose not to do so because we have no evidence that these compounds have similar mechanisms of action and because the lowand high-molecular-weight sums are dominated by MEP and इDEHP metabolite concentrations, respectively.

We examined the association of prenatal phthalate metabolites with repeated continuous (BMI $z$-score, waist circumference z-score, percent body fat) and binary (overweight/obese) outcomes using generalized estimating equations with Gaussian or binomial specification, respectively. Separate models were constructed for each phthalate metabolite. We included interaction terms for metabolite ${ }^{\star}$ visit point to allow the associations to differ by child age. In many cases we have chosen to report results for age 12 years, the oldest age for which we have body size data for this study, because it reflects the longest term impact of phthalate exposure in utero. In subsequent models, we also included interaction terms for metabolite*sex to determine whether associations differed between boys and girls, using the nlcom command in Stata to obtain coefficients for the sex-specific associations at each visit point.

Potential confounders were selected a priori using directed acyclic graphs (Supplementary Figure S1 online). All models controlled for maternal age, education, marital status; mother's years of residence in the United States and family income (below vs. at or above poverty threshold) at the time of pregnancy; smoking during pregnancy; and repeated measures of family food insecurity and child's fast food consumption at each time point. Measures of food insecurity and fast food intake were included because diet, particularly highly processed food and fast food, may be a source of exposure to high-molecularweight phthalates $(25,26)$. We have previously reported associations between childhood BMI and prenatal exposure to bisphenol A (BPA) (27), which-similar to some phthalates-is found in food, plastics, and food containers and was correlated with phthalate concentrations in this data set; thus, all models also controlled for maternal BPA concentrations. All covariates were categorized as shown in Table 2. Because maternal pre-pregnancy BMI, birth weight, and age at onset of puberty might be on the causal pathway between prenatal phthalate exposure and child BMI, we did not include these variables in the main models but did include them in sensitivity analyses. We have previously reported associations of prenatal serum concentrations of the pesticide dichlorodiphenyltrichloroethane (28) and polybrominated diphenyl ether flame retardants (29) with BMI in this cohort. Because we do not have reason to expect these chemicals to be associated with prenatal phthalate exposures and because a significant number of women were missing these variables, we controlled for dichlorodiphenyltrichloroethane and polybrominated diphenyl ether exposure in sensitivity analyses but not in the main models. Associations of prenatal phthalates and body size were very consistent at each time point throughout childhood, and hence, for simplicity, all sensitivity analyses were conducted using models of prenatal exposure and 12-year-old outcomes (the oldest age available), rather than on the repeated-measures models.

In addition, to begin to elucidate the effects of simultaneous exposure to all metabolites measured in this population, we implemented Bayesian Kernel Machine Regression (BKMR) on the continuous outcomes at 12 years, an approach that models the health outcome as a flexible kernel function of the exposure variables, 


\section{Articles | Harley et al.}

Table 2. Demographic characteristics of the study population, CHAMACOS Study, Salinas, CA $(N=345)$

\begin{tabular}{lcc}
\hline & $N$ & $(\%)$ \\
\hline Maternal characteristics at time of pregnancy & & \\
Race/ethnicity & 339 & $(98.3)$ \\
Latina & 3 & $(0.9)$ \\
Non-Latina, White & 3 & $(0.9)$ \\
Other &
\end{tabular}

$\begin{array}{lr}\text { Age (years) } & \\ 18-24 & 149 \\ 25-29 & 111 \\ 30-34 & 55 \\ 35+ & 30\end{array}$

Marital status

Married/living as married

Single

\section{Education \\ $\leqslant 6$ th grade \\ 7-12th grade \\ $\geqslant$ High school graduate}

Years of residence in the USA

$$
\begin{aligned}
& \leqslant 5 \text { years } \\
& 6-10 \text { years } \\
& 11+\text { years } \\
& \text { Entire life }
\end{aligned}
$$

\section{Household income}

Below or equal to poverty

Above poverty

\section{Pre-pregnancy BMI}

Underweight

Normal

Overweight

Obese

\section{Smoked during pregnancy}

No

Yes

\section{Child Characteristics}

Birth weight

$$
\begin{aligned}
& <2,500 \mathrm{~g} \\
& \geqslant 2,500 \mathrm{~g}
\end{aligned}
$$

Table 2. Continued

\begin{tabular}{lrl}
\hline & $N$ & $(\%)$ \\
\hline BMI at age 12 & & \\
Underweight (<5th percentile) & 1 & $(0.3)$ \\
Normal (5th-85th percentile) & 131 & $(40.9)$ \\
Overweight (85th-95th percentile) & 58 & $(18.1)$ \\
Obese ( $>$ 95th percentile) & 130 & $(40.6)$ \\
& & \\
Food insecurity at age 12 & & \\
Food secure & 224 & $(71.6)$ \\
Insecure without hunger & 70 & $(22.4)$ \\
Insecure with hunger & 19 & $(6.1)$ \\
& & \\
Fast food consumption at age 12 & & \\
$\quad<1$ time per week & 97 & $(30.9)$ \\
1-2 times per week & 206 & $(65.6)$ \\
3+ times per week & 11 & $(3.5)$ \\
\hline
\end{tabular}

adjusted for covariates (30). The phthalate metabolite concentrations were all moderately correlated with each other $(r=0.18-0.71)$, precluding their inclusion in the same regression model, but making them good candidates for BKMR. We used BKMR as an approach for variable selection to determine which phthalate concentration variables are influential enough to include in the analysis and to determine the influence of each exposure variable on the outcome when other variables are held at specified values. Although BKMR can also examine interaction effects, this was beyond the scope of the present analysis.

All regression analyses were performed using Stata 13 (College Station, Texas), and BKMR analyses were conducted using the BKMR package in R (Vienna, Austria).

\section{RESULTS}

Mothers in the study tended to be Latinas of low-income and low-education level (high-school education or less) (Table 2). Most women were married or living as married, under the age of 30 years, and had been living in the United States for 10 years or less at the time of their pregnancy. More than $65 \%$ of mothers were overweight or obese before pregnancy and $58.5 \%$ of their children were overweight or obese by 12 years of age. Mothers whose children were not included in the analysis (i.e., did not have phthalate measurements and at least one anthropometric measurement) were younger, more likely to live in the United States for less than 5 years, and were less likely to be obese (Supplementary Table S1). Mean BMI, waist circumference, and percent body fat for boys and girls at each age are shown in Supplementary Table S2. BMI measurements across ages 5-12 years were highly and significantly correlated. The lowest correlation, between BMI at 5 and that at 12 years of age, was 0.78. (Data not shown.)

The distribution of urinary phthalate metabolites during pregnancy is shown in Table 3. Phthalate metabolites were detected in most women (range: $87.5-100 \%$ detection), with 
Table 3. Distribution of uncorrected and specific-gravity-corrected (italics) phthalate metabolites in maternal urine collected at two time points during pregnancy $(N=345)$

\begin{tabular}{|c|c|c|c|c|c|c|c|c|c|}
\hline \multirow[b]{2}{*}{ Phthalate metabolite } & \multicolumn{2}{|c|}{$\%>$ LOD } & \multicolumn{7}{|c|}{ Average of two measurements } \\
\hline & 1st measurement ${ }^{a}$ & 2nd measurement ${ }^{b}$ & Geo. mean & 10th\% & 25 th $\%$ & 50 th $\%$ & 75th\% & 90th\% & 95th\% \\
\hline \multirow[t]{2}{*}{ MEP (ng/ml) } & $100.0 \%$ & $99.5 \%$ & 183.7 & 34.4 & 80.3 & 185.0 & 413.0 & 795.5 & $1,280.0$ \\
\hline & & & 236.0 & 47.7 & 106.0 & 231.0 & 500.3 & 953.5 & $1,620.4$ \\
\hline \multirow[t]{2}{*}{ MBP (ng/ml) } & $98.4 \%$ & $100.0 \%$ & 22.8 & 6.4 & 12.2 & 22.1 & 45.5 & 80.0 & 107.3 \\
\hline & & & 28.5 & 9.1 & 15.9 & 27.5 & 51.7 & 93.4 & 128.1 \\
\hline \multirow[t]{2}{*}{$\operatorname{MiBP}(\mathrm{ng} / \mathrm{ml})$} & $92.8 \%$ & $95.0 \%$ & 2.7 & 0.8 & 1.5 & 2.8 & 5.4 & 9.5 & 16.5 \\
\hline & & & 3.4 & 1.1 & 1.9 & 3.5 & 6.5 & 11.0 & 16.3 \\
\hline \multirow[t]{2}{*}{ MBzP (ng/ml) } & $97.2 \%$ & $98.8 \%$ & 7.3 & 1.7 & 3.8 & 8.0 & 15.4 & 26.5 & 35.1 \\
\hline & & & 9.1 & 2.5 & 4.9 & 9.5 & 18.4 & 29.4 & 44.1 \\
\hline \multirow[t]{2}{*}{$\sum \mathrm{DEHP}(\mathrm{nmol} / \mathrm{ml})$} & NA & NA & 0.2 & 0.1 & 0.1 & 0.2 & 0.3 & 0.6 & 0.8 \\
\hline & & & 0.2 & 0.1 & 0.1 & 0.2 & 0.4 & 0.6 & 0.9 \\
\hline \multirow[t]{2}{*}{ MEHP (ng/ml) } & $88.0 \%$ & $91.4 \%$ & 3.6 & 1.0 & 2.1 & 3.7 & 7.0 & 12.8 & 20.8 \\
\hline & & & 4.5 & 1.4 & 2.6 & 4.4 & 7.6 & 15.7 & 20.3 \\
\hline \multirow[t]{2}{*}{ MEHHP (ng/ml) } & $100.0 \%$ & $99.3 \%$ & 15.1 & 4.5 & 8.6 & 15.2 & 27.8 & 49.1 & 72.8 \\
\hline & & & 18.8 & 6.4 & 11.3 & 18.4 & 32.9 & 55.3 & 75.7 \\
\hline \multirow[t]{2}{*}{ MECPP (ng/ml) } & $100.0 \%$ & $100.0 \%$ & 25.9 & 9.1 & 15.7 & 25.2 & 43.1 & 70.2 & 92.8 \\
\hline & & & 32.4 & 12.9 & 21.0 & 31.1 & 50.5 & 78.3 & 110.3 \\
\hline \multirow[t]{2}{*}{ MEOHP (ng/ml) } & $98.2 \%$ & $98.6 \%$ & 11.2 & 3.4 & 6.6 & 11.2 & 20.8 & 35.4 & 51.1 \\
\hline & & & 13.8 & 5.0 & 8.0 & 14.1 & 22.6 & 39.0 & 53.6 \\
\hline \multirow[t]{2}{*}{ MCPP (ng/ml) } & $88.0 \%$ & $93.5 \%$ & 1.7 & 0.6 & 1.0 & 2.1 & 3.2 & 4.9 & 6.6 \\
\hline & & & 2.2 & 0.8 & 1.4 & 2.4 & 3.8 & 5.3 & 7.6 \\
\hline \multirow[t]{2}{*}{ MCOP (ng/ml) } & $96.8 \%$ & $96.6 \%$ & 3.0 & 1.0 & 1.7 & 3.2 & 5.0 & 7.5 & 11.0 \\
\hline & & & 3.7 & 1.5 & 2.4 & 3.9 & 5.5 & 8.9 & 12.2 \\
\hline \multirow[t]{2}{*}{ MCNP (ng/ml) } & $95.1 \%$ & $96.7 \%$ & 1.8 & 0.6 & 1.2 & 1.9 & 0.3 & 4.5 & 6.2 \\
\hline & & & 2.3 & 0.9 & 1.5 & 2.3 & 3.5 & 5.5 & 7.6 \\
\hline
\end{tabular}

LOD, limit of detection.

${ }^{\mathrm{a}}$ Mean \pm s.d. $=14.0 \pm 5.0$ weeks gestation.

${ }^{b}$ Mean \pm s.d. $=26.9 \pm 2.5$ weeks gestation.

MEP exhibiting considerably higher concentrations than the other phthalate metabolites. Analyte concentrations were similar to those measured in women of reproductive age in the NHANES data set for a similar time period (1999-2000 or 2001-2002) (31).

Table 4 shows the association of prenatal phthalate metabolites with odds of being overweight or obese at $5,7,9,10^{1 / 2}$, and 12 years of age, by all children together and separately for boys and girls. We saw no evidence of interaction by sex for any metabolites except MBP ( $P$ value for sex interaction $=$ $0.04)$. For MEP, MBzP, and $\Sigma$ DEHP, we observed a consistent $20-30 \%$ increase in odds of overweight/obesity with each doubling of prenatal urinary metabolite concentration at each age point in both boys and girls. For MBP, we observed a $30-40 \%$ increase in odds in boys only (Table 4 ).

Table 5 shows the associations of phthalate metabolite concentrations with BMI z-score, waist circumference $z$-score, and percent body fat as continuous variables. Results were very similar in boys and girls (Supplementary Table S3), and hence results are shown for all children combined. We observed consistent positive associations of MEP with BMI, waist circumference, and percent body fat at each time point. At age 12 years, each doubling in urinary MEP concentrations was associated with increases in BMI $z$-score $(\beta=0.09,95 \%$ confidence interval (CI): 0.04, 0.15), waist circumference $z$-score $(\beta=0.10,95 \% \mathrm{CI}: 0.04,0.15)$, and percent body fat ( $\beta=1.0,95 \%$ CI: $0.2,1.6)$, and results were similar at younger ages. We also observed positive associations with other metabolites, including $\mathrm{MBP}, \mathrm{MiBP}, \mathrm{MBzP}$, and $\Sigma \mathrm{DEHP}$, with BMI, waist circumference, and percent body fat (Table 5), but these associations were less consistent over time and were stronger at younger ages. Only MBP and MCPP showed some evidence of sex interaction (sex interaction $P$ values $<0.1$ ) in both cases, with positive associations seen for boys and associations close to null for girls (Supplementary Table S3). For example, at age 12 years, each doubling of MBP was associated with a change in BMI $z$ score of 0.13 ( $95 \%$ CI: $0.02,0.24)$ in boys vs. -0.01 (95\% CI: $-0.14,0.12)$ in girls (sex interaction $P$ value $=0.08)$ and a change in waist circumference $z$-score of 0.10 (95\% CI: 0.01 , 


\section{Articles | Harley et al.}

Table 4. Association of $\log _{2}$ prenatal urinary phthalate metabolite concentrations with odds of overweight/obesity in all children and separately by boys and girls ( $N=341$ children, 1,552 observations)

\begin{tabular}{|c|c|c|c|c|c|c|}
\hline & 5 year & 7 year & 9 year & 10.5 year & 12 year & Sex interaction \\
\hline & OR $(95 \% \mathrm{Cl})$ & OR $(95 \% \mathrm{Cl})$ & OR $(95 \% \mathrm{Cl})$ & OR $(95 \% \mathrm{Cl})$ & OR $(95 \% \mathrm{Cl})$ & $P$ value \\
\hline \multicolumn{7}{|l|}{ MEP } \\
\hline Boys & $1.3(1.1,1.5)^{*}$ & $1.3(1.1,1.5)^{*}$ & $1.3(1.1,1.5)^{*}$ & $1.3(1.1,1.5)^{*}$ & $1.3(1.1,1.5)^{*}$ & \\
\hline Girls & $1.3(1.1,1.5)^{*}$ & $1.3(1.1,1.5)^{*}$ & $1.3(1.1,1.5)^{*}$ & $1.3(1.1,1.5)^{\dagger}$ & $1.3(1.0,1.5)^{\dagger}$ & 0.93 \\
\hline Boys & $1.3(1.1,1.7)^{\dagger}$ & $1.3(1.1,1.6)^{*}$ & $1.4(1.1,1.7)^{*}$ & $1.4(1.1,1.7)^{*}$ & $1.4(1.1,1.8)^{*}$ & \\
\hline Girls & $1.0(0.7,1.2)$ & $1.0(0.8,1.2)$ & $1.0(0.8,1.2)$ & $1.0(0.8,1.2)$ & $1.0(0.8,1.3)$ & $0.04^{\dagger}$ \\
\hline \multicolumn{7}{|l|}{ MiBP } \\
\hline All & $1.1(0.9,1.3)$ & $1.1(0.9,1.2)$ & $1.1(1.0,1.3)$ & $1.1(0.9,1.3)$ & $1.1(1.0,1.3)$ & \\
\hline All & $1.2(1.0,1.4)^{\ddagger}$ & $1.2(1.0,1.4)^{\ddagger}$ & $1.2(1.0,1.4)^{\dagger}$ & $1.2(1.0,1.4)^{\ddagger}$ & $1.2(1.0,1.4)^{\ddagger}$ & \\
\hline Boys & $1.2(1.0,1.5)$ & $1.2(1.0,1.5)$ & $1.2(1.0,1.5)^{\ddagger}$ & $1.2(1.0,1.5)$ & $1.2(1.0,1.5)^{\ddagger}$ & \\
\hline Girls & $1.1(0.9,1.4)$ & $1.1(0.9,1.4)$ & $1.2(0.9,1.4)$ & $1.1(0.9,1.4)$ & $1.2(0.9,1.5)$ & 0.81 \\
\hline \multicolumn{7}{|l|}{$\Sigma \mathrm{DEHP}$} \\
\hline All & $1.1(0.9,1.4)$ & $1.2(1.0,1.5)^{\ddagger}$ & $1.2(1.0,1.5)^{\ddagger}$ & $1.2(1.0,1.5)^{\ddagger}$ & $1.3(1.0,1.6)^{\dagger}$ & \\
\hline Boys & $1.1(0.8,1.6)$ & $1.2(0.9,1.7)$ & $1.2(0.9,1.6)$ & $1.3(0.9,1.7)$ & $1.3(1.0,1.7)$ & \\
\hline Girls & $1.1(0.8,1.5)$ & $1.2(0.9,1.6)$ & $1.2(0.9,1.6)$ & $1.2(0.9,1.6)$ & $1.3(0.9,1.7)$ & 0.89 \\
\hline \multicolumn{7}{|l|}{ MCPP } \\
\hline All & $1.0(0.8,1.2)$ & $1.0(0.8,1.2)$ & $1.0(0.8,1.2)$ & $1.0(0.8,1.2)$ & $1.1(0.9,1.3)$ & \\
\hline \multicolumn{7}{|l|}{ MCNP } \\
\hline All & $1.2(1.0,1.6)^{\dagger}$ & $1.2(1.0,1.5)$ & $1.1(0.9,1.4)$ & $1.1(0.9,1.4)$ & $1.1(0.8,1.4)$ & \\
\hline Boys & $1.4(1.0,1.9)^{\dagger}$ & $1.4(1.0,1.8)^{\dagger}$ & $1.3(1.0,1.7)^{\ddagger}$ & $1.2(0.9,1.7)$ & $1.2(0.9,1.7)$ & \\
\hline Girls & $1.2(0.9,1.5)$ & $1.1(0.9,1.5)$ & $1.1(0.8,1.4)$ & $1.0(0.8,1.4)$ & $1.0(0.8,1.4)$ & 0.33 \\
\hline
\end{tabular}

Separate models created for each phthalate metabolite or sum of metabolites ( $\left.\sum D E H P\right) ;$ coefficients for boys and girls obtained from model with sex interaction term.

Models control for maternal age, maternal education, marital status, years in United States prior to delivery, smoking during pregnancy, poverty status during pregnancy, child's food insecurity at each time point, child's fast food consumption at each time point, and $\log _{2}$ prenatal BPA.

${ }^{*} P<0.01$.

${ }^{+} p<0.05$.

${ }^{\ddagger} p<0.10$.

0.20 ) in boys vs. -0.03 (95\% CI: $-0.15,0.08)$ in girls (sex interaction $P$ value $=0.08)$.

Similar results were seen when phthalate metabolites were examined as categorical variables by quartiles at age 12 years (Figure 1). Being in the highest concentration quartile of prenatal phthalate biomarkers was associated with increased odds of overweight/obesity for MEP (odds ratio $(\mathrm{OR})=2.0$, 95\% confidence interval (CI): 1.0, 3.9), MBP (OR $=2.1,95 \%$ CI: $1.1,4.2), \mathrm{MBzP}(\mathrm{OR}=1.9,95 \% \mathrm{CI}: 0.9,3.7)$, and $\Sigma \mathrm{DEHP}$
$(\mathrm{OR}=2.2,95 \% \mathrm{CI}: 1.1,4.5)$. There was no indication of sex interaction for overweight/obesity at age 12 when phthalate metabolites were considered as categorical variables, and hence the results are for boys and girls combined.

In sensitivity analyses, associations with outcomes at age 12 were very similar when we additionally controlled for maternal pre-pregnancy BMI, birth weight, or age at onset of puberty (Supplementary Table S4). Results were virtually unchanged when we additionally controlled for 


\section{Prenatal phthalates and childhood obesity $\mid$ Articles}

Table 5. Association of $\log _{2}$ prenatal urinary phthalate metabolite concentrations with BMI $z$-score, waist circumference $z$-score, and percent body fat in children at various ages

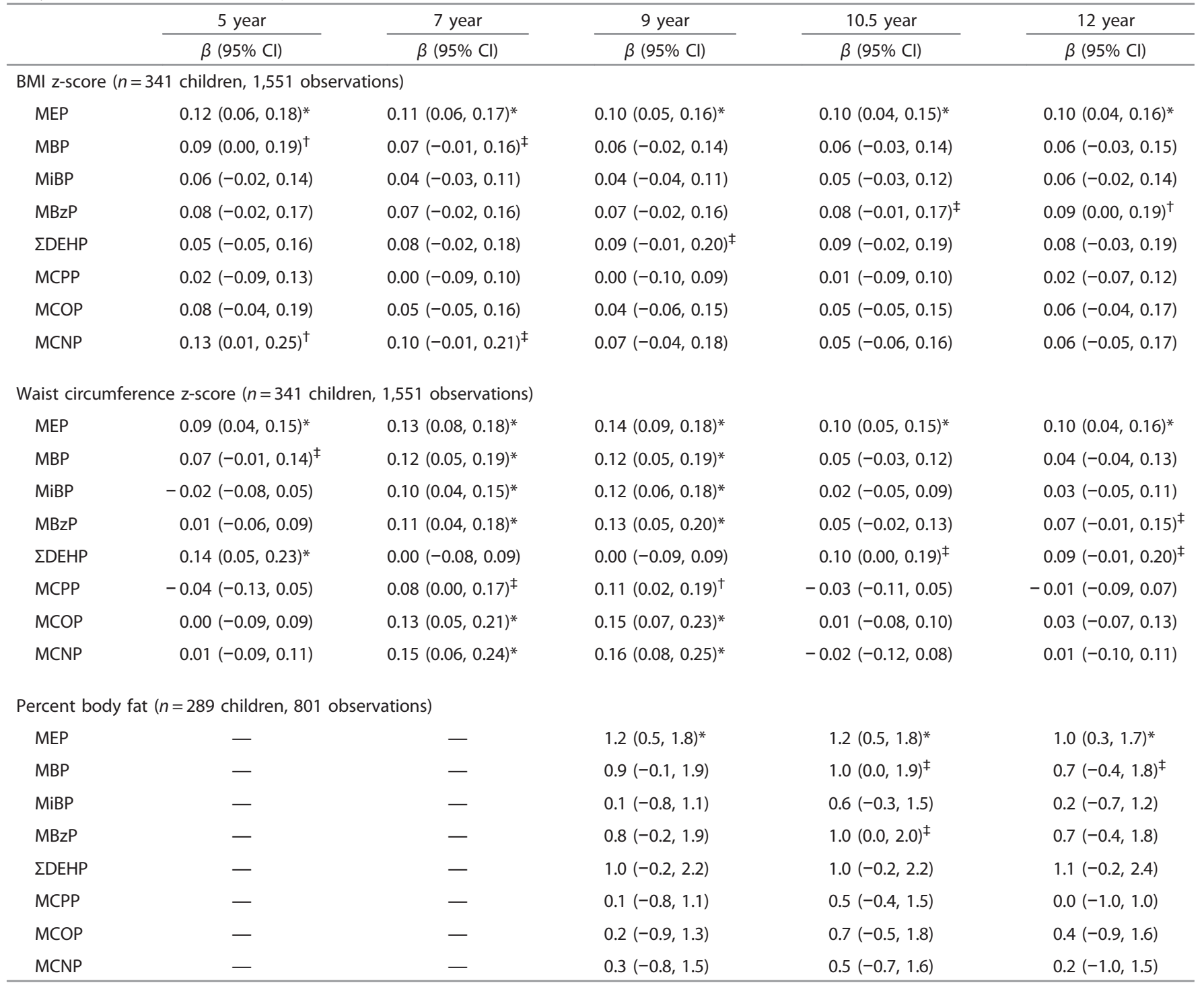

Separate models created for each phthalate metabolite or sum of metabolites ( $\Sigma \mathrm{DEHP})$.

Models control for maternal age, maternal education, marital status, years in United States prior to delivery, smoking during pregnancy, poverty status during pregnancy, child's

food insecurity at each time point, child's fast food consumption at each time point, and $\log _{2}$ prenatal BPA.

${ }^{*} P<0.01$.

${ }^{\dagger} p<0.05$.

${ }^{\ddagger} p<0.10$.

dichlorodiphenyltrichloroethane or polybrominated diphenyl ethers (not shown).

The BKMR analysis of simultaneous phthalate exposure identified all phthalate metabolite variables as important contributors to BMI $z$-score. Figure 2 illustrates that the trend of positive associations of MEP, MBP, MBzP, and $\Sigma \mathrm{DEHP}$ with $\mathrm{BMI} z$-score at age 12 observed in the single metabolite linear regression models persists in the BKMR models including all other phthalates held at their median values, and also controlling for other covariates. Both MCPP and MCOP demonstrated negative associations with BMI $z$-score that were not apparent in the linear regression models, although in the case of MCPP this was strongly influenced by sparse data at rather low values. No other phthalate metabolites showed a remarkable change in slope.

\section{DISCUSSION}

We found that higher concentrations of urinary MEP, MBzP, and $\Sigma$ DEHP metabolites in pregnant mothers were associated with increased odds of their children being overweight or obese between 5 and 12 years of age. Higher prenatal MEP concentrations were consistently associated with increased BMI, waist circumference, and percent body fat in children between 5 and 12 years of age. Higher prenatal MBP, MBzP, and $\Sigma$ DEHP concentrations were also associated with larger body size over the same time period but less consistently. 


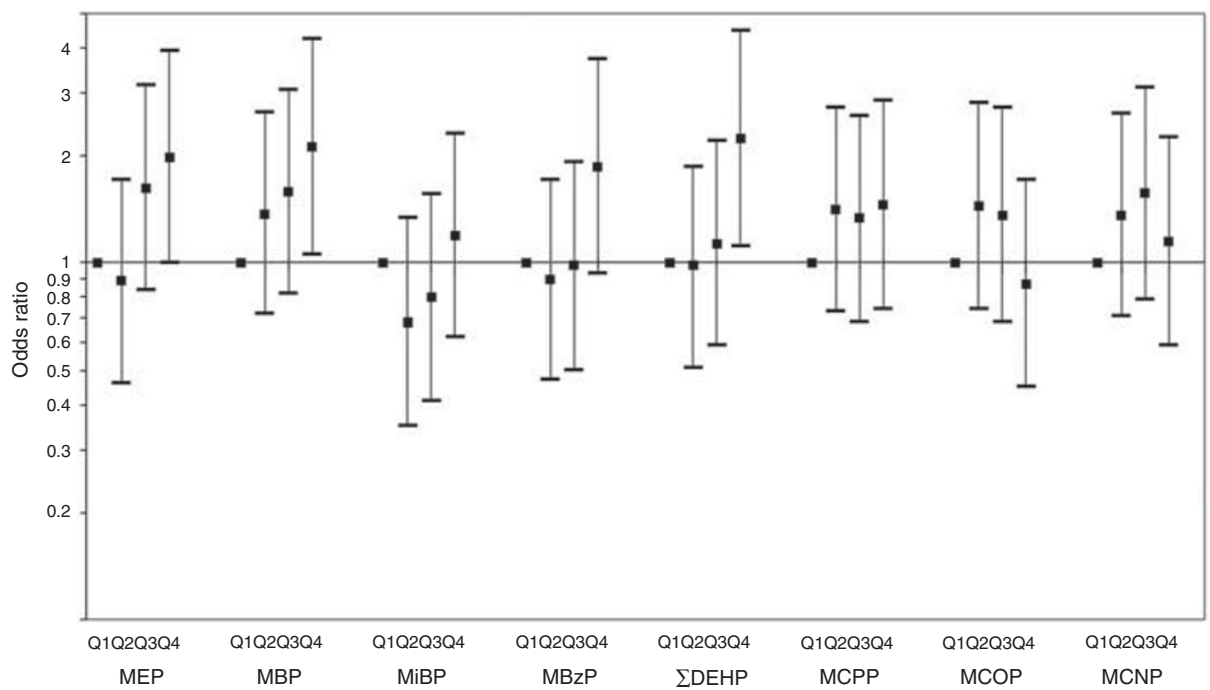

Figure 1. Adjusted odds ratios of overweight/obesity at age 12 years comparing maternal quartile of urinary phthalate metabolites during pregnancy.
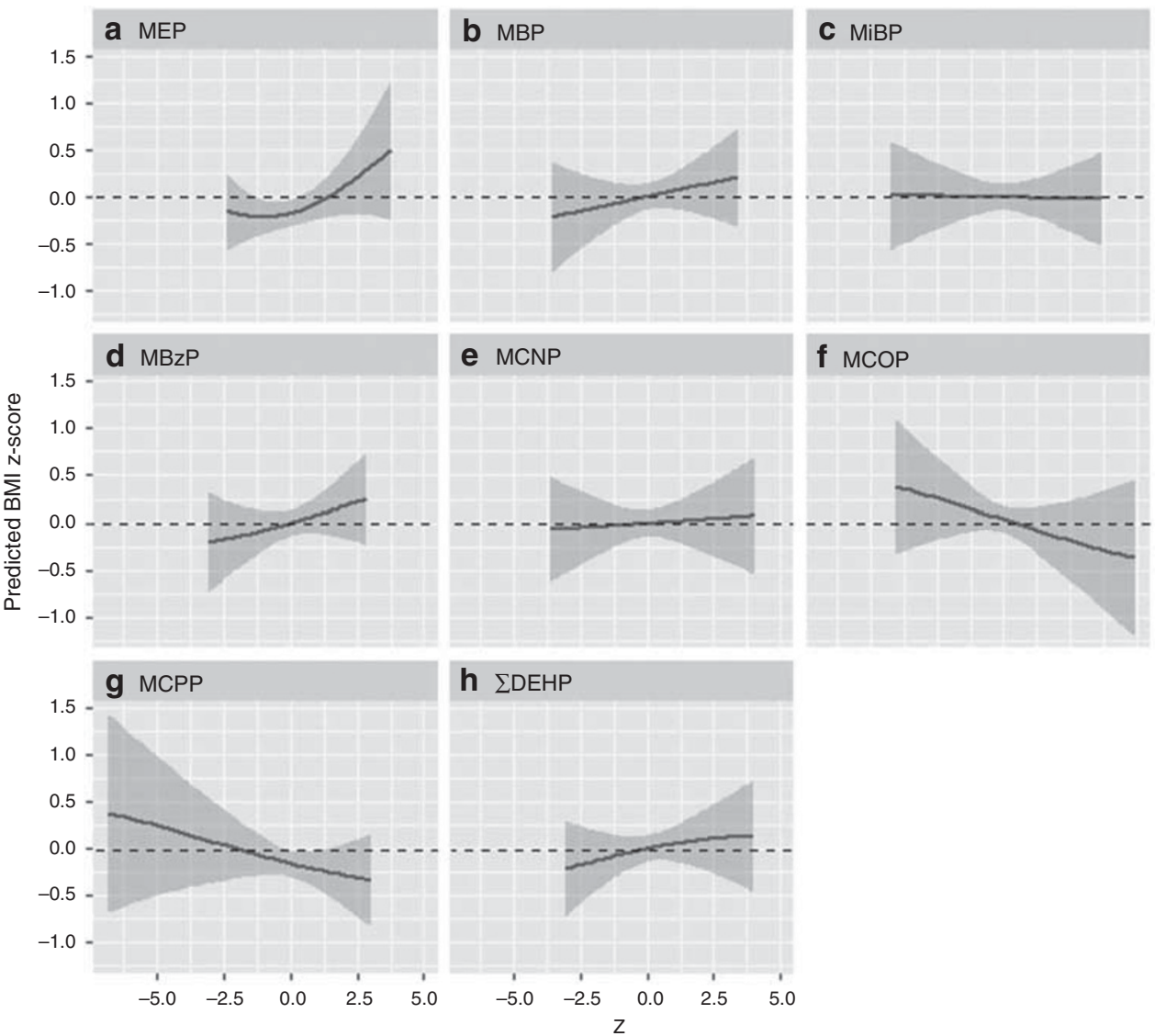

Figure 2. Adjusted associations and $95 \%$ credible intervals for concentrations of MEP (a), MBP (b), MiBP (c), MBzP (d), MCNP (e), MCOP (f), MCPP (g), and $\sum$ DEHP metabolites (h) with BMI $z$-score at age 12, holding all other phthalate metabolite concentrations at their median.

Results were similar at multiple ages between 5 and 12 years of age, suggesting that onset of puberty did not affect our findings. In addition, unlike previous studies, our results were quite similar between boys and girls. We saw some evidence of sex interaction with MBP and MCPP, with boys showing stronger positive associations with body size than girls, but not with other metabolites.

Five other investigations (13-17) have used similar study designs as ours, measuring phthalate metabolites in prenatal urine and body weight in childhood, although no other study 
followed up children beyond 7 years of age. Our findings are consistent with those of Botton et al. (17), who reported that prenatal urinary concentrations of MEP were associated with increased weight growth velocity at ages 2 and 4 years and increased BMI at 5 years in French boys $(N=520)$. The study by Botton et al. did not include girls and could not examine interaction by sex. Like Botton et al., we found associations of prenatal MEP with increased BMI at 5 years of age; we also found that these associations persisted through age 12 . However, our findings are not consistent with three other studies that have found different prenatal phthalate metabolites to be associated with decreased BMI in boys (14-16) or with the pooled study that found increased odds of overweight/obesity with MCPP and decreased odds of overweight/ obesity with MEP and $\Sigma$ DEHP in girls (13).

Reasons for the inconsistency of study findings may relate to the difficulty in characterizing ongoing phthalate exposure during pregnancy. Phthalates are non-persistent compounds with short biological half-lives that are rapidly excreted from the body (32); thus, urinary metabolites reflect only recent exposure. Previous studies have shown that spot urine measures of phthalate metabolites have weak to moderate temporal reliability with interclass correlation coefficients often ranging from $\sim 0.2$ to 0.5 (33), which is consistent with our study. With the exception of the study by Valvi et al. (16), the previous studies assessed exposure during pregnancy with a single spot urine sample, which may not have been adequate to characterize phthalate exposure throughout pregnancy. In the current study, we averaged measures in two pregnancy urine samples to better characterize ongoing exposure, although this too may be insufficient to account for the temporal variability across pregnancy.

Another source of inconsistency with previous studies may relate to timing of exposure. Although we measured phthalate exposure in the prenatal period, we have no information about phthalate exposure during childhood, which may also be relevant to later risk for obesity. Our study population also differs from those of previous studies in terms of demographics. The vast majority of mothers in our study were recent immigrants from Mexico who were of low income and did not complete high school. These unique demographics may affect the generalizability of our results.

It is also possible that previous studies assessed BMI too early in childhood to detect associations with later obesity. On the basis of animal studies that only observed increased fat mass in offspring after the animals reached sexual maturity $(9,10)$, we hypothesized that we might not detect associations between phthalate exposure and body size until age 12 or later. However, the fact that we found consistent positive associations of phthalate metabolites with BMI, waist circumference, percent body fat, and overweight/obesity consistently, beginning at age 5 , argues against this hypothesis and suggests that puberty did not play a strong role in this association.

Possible biological mechanisms for our observed associations of several phthalates with increased BMI and risk for obesity include PPAR activation, adipocyte differentiation, and thyroid hormone disruption. MEHP, MBzP, and MBP activate PPAR- $\alpha$ and PPAR- $\gamma$ nuclear receptors, key regulators of adipogenesis and energy storage, in both rodent and human cell lines (34). MEHP has been shown to stimulate lipid accumulation in differentiating adipocytes in vitro via PPAR- $\gamma$ activation, leading to increased release of leptin, resistin, and adiponectin (35). In addition, DEHP has been shown to induce hypothyroidism in mice, leading to energy metabolism imbalance and increased white adipose tissue (8). In epidemiologic studies, several phthalate metabolites are also negatively associated with thyroid hormone levels (36), which could impact the regulation of resting energy expenditure and increase body weight. Various phthalates have demonstrated anti-androgenic or weak estrogenic properties (37), which can also impact fat quantity and distribution (38).

Exposure to the phthalates linked to obesity in this study is pervasive. Between 2001 and 2010, almost all Americans sampled in NHANES had detectable concentrations of metabolites of DEHP (e.g., MEOHP, MEHHP, MECCP), DEP (i.e., MEP), and DBP (i.e., MBP) in their urine (6). The primary routes of exposure to the high-molecular-weight phthalates used in plastics, including DEHP, DiNP, and BBzP, appear to be via diet (39), possibly a result of contact with plastics during food processing, packaging, and storage (40). A recent study found higher urinary metabolites of DEHP among individuals consuming more fast food (41), highlighting the difficulty in separating the effects of diet vs. high-molecularweight phthalates on obesity. In contrast, the main sources of exposure to DEP and DBP are thought to be personal care product use and some medications (42-44); thus, diet is less likely to be a confounding factor in these associations. We found associations of prenatal exposure to DEP, DBP, BBzP, and DEHP with increased BMI, waist circumference, percent body fat, and increased risk of being overweight or obese in late childhood. These associations persisted when the joint impact of all phthalates was considered. This is the first study to demonstrate these associations, and findings will need to be replicated in other populations. Future studies should assess the trajectory of body size over time to determine in greater detail whether an increased BMI at a young age stays consistent throughout childhood.

\section{SUPPLEMENTARY MATERIAL}

Supplementary material is linked to the online version of the paper at http://www.nature.com/pr

\section{ACKNOWLEDGMENTS}

We thank the laboratory and field staff and participants of the CHAMACOS study for their contributions. We also thank Xiaoyun Ye, Manori Silva, Ella Samandar, Jim Preau and Tao Jia at CDC for technical assistance in measuring the urinary concentrations of phthalate metabolites.

\section{STATEMENT OF FINANCIAL SUPPORT}

This publication was supported by grants PO1 ES009605 and RO1 ES021369 from the National Institute of Environmental Health Sciences (NIEHS) and grants R82670901 and RD83451301 from the US Environmental Protection Agency (EPA). 


\section{Articles | Harley et al.}

Disclosure: The findings and conclusions in this report are those of the authors and do not necessarily represent the official position of the Centers for Disease Control and Prevention. Use of trade names is for identification only and does not imply endorsement by the CDC, the Public Health Service, or the US Department of Health and Human Services.

\section{REFERENCES}

1. Ogden C Prevalence of obesity among children and adolescents: United States, trends 1963-1965 through 2007-2008. Center Dis Control Prevent Natl Center Health Statistics. 2010;201:1-5.

2. Flegal KM, Kruszon-Moran D, Carroll MD, Fryar CD, Ogden CL. Trends in obesity among adults in the United States, 2005 to 2014. JAMA 2016;315:2284-91.

3. Baillie-Hamilton PF. Chemical toxins: a hypothesis to explain the global obesity epidemic. J Altern Complement Med 2002;8:185-92.

4. Thayer KA, Heindel JJ, Bucher JR, Gallo MA. Role of environmental chemicals in diabetes and obesity: a National Toxicology Program workshop review. Environ Health Perspect 2012;120:779-89.

5. Janesick A, Blumberg B. Endocrine disrupting chemicals and the developmental programming of adipogenesis and obesity. Birth Defects Res C Embryo Today 2011;93:34-50.

6. Zota AR, Calafat AM, Woodruff TJ. Temporal trends in phthalate exposures: findings from the National Health and Nutrition Examination Survey, 2001-2010. Environ Health Perspect 2014;122:235-41.

7. Desvergne B, Feige JN, Casals-Casas C. PPAR-mediated activity of phthalates: a link to the obesity epidemic? Mol Cell Endocrinol 2009;304: 43-8.

8. Lv Z, Cheng J, Huang S, et al. DEHP induces obesity and hypothyroidism through both central and peripheral pathways in $\mathrm{C} 3 \mathrm{H} / \mathrm{He}$ mice. Obesity 2016;24:368-78.

9. Schmidt JS, Schaedlich K, Fiandanese N, Pocar P, Fischer B. Effects of di (2-ethylhexyl) phthalate (DEHP) on female fertility and adipogenesis in C3H/N mice. Environ Health Perspect 2012;120:1123-9.

10. Strakovsky RS, Lezmi S, Shkoda I, Flaws JA, Helferich WG, Pan YX. In utero growth restriction and catch-up adipogenesis after developmental di (2-ethylhexyl) phthalate exposure cause glucose intolerance in adult male rats following a high-fat dietary challenge. J Nutr Biochem 2015;26: 1208-20.

11. Trasande L, Attina TM, Sathyanarayana S, Spanier AJ, Blustein J. Race/ ethnicity-specific associations of urinary phthalates with childhood body mass in a nationally representative sample. Environ Health Perspect (Online) 2013;121:501.

12. Hatch EE, Nelson JW, Qureshi MM, et al. Association of urinary phthalate metabolite concentrations with body mass index and waist circumference: a cross-sectional study of NHANES data, 1999-2002. Environ Health 2008;7:27.

13. Buckley JP, Engel SM, Braun JM, et al. Prenatal phthalate exposures and body mass index among 4-to 7-year-old children: a pooled analysis. Epidemiology 2016;27:449-58.

14. Buckley JP, Engel SM, Mendez MA, et al. Prenatal phthalate exposures and childhood fat mass in a New York City cohort. Environ Health Perspect (Online) 2016;124:507.

15. Maresca MM, Hoepner LA, Hassoun A, et al. Prenatal exposure to phthalates and childhood body size in an urban cohort. Environ Health Perspect 2016;124:514-20.

16. Valvi D, Casas M, Romaguera D, et al. Prenatal phthalate exposure and childhood growth and blood pressure: evidence from the Spanish INMA-Sabadell birth cohort study. Environ Health Perspect 2015;123: $1022-9$.

17. Botton J, Philippat C, Calafat AM, et al. Phthalate pregnancy exposure and male offspring growth from the intra-uterine period to five years of age. Environ Res. 2016;151:601-9.

18. Deierlein AL, Wolff MS, Pajak A, et al. Longitudinal associations of phthalate exposures during childhood and body size measurements in young girls. Epidemiology 2016;27:492-9.
19. Silva MJ, Samandar E, Preau JL Jr, Reidy JA, Needham LL, Calafat AM. Quantification of 22 phthalate metabolites in human urine. J Chromatogr 2007;860:106-2.

20. Lubin JH, Colt JS, Camann D, et al. Epidemiologic evaluation of measurement data in the presence of detection limits. Environ Health Perspect 2004;112:1691-6.

21. Mahalingaiah S, Meeker JD, Pearson KR, et al. Temporal variability and predictors of urinary bisphenol A concentrations in men and women. Environ Health Perspect 2008;116:173-8.

22. National Center for Health Statistics CDC Growth Charts, United States 2005. https://www.cdc.gov/growthcharts/percentile_data_files.htm.

23. Kuczmarski RJ, Ogden CL, Guo SS, et al. 2000 CDC Growth Charts for the United States: methods and development. Vital Health Stat 11 2002;246: $1-190$.

24. Koch HM, Bolt HM, Preuss R, Angerer J. New metabolites of di(2ethylhexyl)phthalate (DEHP) in human urine and serum after single oral doses of deuterium-labelled DEHP. Arch Toxicol 2005;79:367-76.

25. Schecter A, Lorber M, Guo Y, et al. Phthalate concentrations and dietary exposure from food purchased in New York State. Environ Health Persp (Online) 2013;121:473.

26. Rudel RA, Gray JM, Engel CL, et al. Food packaging and bisphenol A and bis(2-ethyhexyl) phthalate exposure: findings from a dietary intervention. Environ Health Perspect 2011;119:914-20.

27. Harley KG, Aguilar Schall R, Chevrier J, et al. Prenatal and postnatal bisphenol A exposure and body mass index in childhood in the CHAMACOS cohort. Environ Health Perspect 2013;121:514-20.

28. Warner M, Wesselink A, Harley KG, Bradman A, Kogut K, Eskenazi B. Prenatal exposure to dichlorodiphenyltrichloroethane and obesity at 9 years of age in the CHAMACOS study cohort. Am J Epidemiol 2014;179:1312-22.

29. Erkin-Cakmak A1, Harley KG, Chevrier J, Bradman A, Kogut K, Huen K, Eskenazi B. In utero and childhood polybrominated diphenyl ether exposures and body mass at age 7 years: the CHAMACOS study. Environ Health Perspect 2015;123:636-42.

30. Bobb JF, Valeri L, Claus Henn B, et al. Bayesian kernel machine regression for estimating the health effects of multi-pollutant mixtures. Biostatistics 2015;16:493-508.

31. CDC. Fourth Report on Human Exposure to Environmental Chemicals. US Department of Health and Human Services. Centers for Disease Control and Prevention: Atlanta, GA, 2009.

32. Janjua NR, Frederiksen H, Skakkebaek NE, Wulf HC, Andersson AM. Urinary excretion of phthalates and paraben after repeated whole-body topical application in humans. Int J Androl 2008;31:118-30.

33. Johns LE, Cooper GS, Galizia A, Meeker JD. Exposure assessment issues in epidemiology studies of phthalates. Environ Int 2015;85:27-39.

34. Hurst CH, Waxman DJ. Activation of PPARalpha and PPARgamma by environmental phthalate monoesters. Toxicol Sci 2003;74:297-308.

35. Taxvig C, Dreisig K, Boberg J, et al. Differential effects of environmental chemicals and food contaminants on adipogenesis, biomarker release and PPARgamma activation. Mol Cell Endocrinol 2012;361:106-5.

36. Boas M, Frederiksen H, Feldt-Rasmussen U, et al. Childhood exposure to phthalates: associations with thyroid function, insulin-like growth factor I, and growth. Environ Health Perspect 2010;118:1458-64.

37. Takeuchi S, Iida M, Kobayashi S, Jin K, Matsuda T, Kojima H. Differential effects of phthalate esters on transcriptional activities via human estrogen receptors alpha and beta, and androgen receptor. Toxicology 2005;210:223-33.

38. Newbold RR. Perinatal exposure to endocrine disrupting chemicals with estrogenic activity and the development of obesity. Robert H Lustig (ed). In: Obesity Before Birth. Springer: New York, 2011, pp 367-82.

39. Koch HM, Lorber M, Christensen KL, Palmke C, Koslitz S, Bruning T. Identifying sources of phthalate exposure with human biomonitoring: results of a $48 \mathrm{~h}$ fasting study with urine collection and personal activity patterns. Int J Hyg Environ Health 2013;216:672-81.

40. Serrano SE, Braun J, Trasande L, Dills R, Sathyanarayana S. Phthalates and diet: a review of the food monitoring and epidemiology data. Environ Health 2014;13:43. 
41. Zota AR, Phillips CA, Mitro SD. Recent fast food consumption and bisphenol A and phthalates exposures among the U.S. population in NHANES, 2003-2010. Environ Health Perspect 2016;124: $1521-8$.

42. Hernandez-Diaz S, Mitchell AA, Kelley KE, Calafat AM, Hauser R. Medications as a potential source of exposure to phthalates in the U.S. population. Environ Health Perspect 2009;117:185-9.
43. Guo Y, Kannan K. A survey of phthalates and parabens in personal care products from the United States and its implications for human exposure. Environ Sci Technol 2013;47:14442-9.

44. Harley KG, Kogut K, Madrigal DS, et al. Reducing phthalate, paraben, and phenol exposure from personal care products in adolescent girls: findings from the HERMOSA Intervention Study. Environ Health Perspect 2016;124:1600-7. 